\title{
Hochschulisches Lernen - eine analytische Perspektive
}

\author{
Rüdiger Rhein
}

Eingegangen: 22. März 2015 / Angenommen: 30. Oktober 2015 / Online publiziert: 15. Dezember 2015

(C) Die Autor(en) 2015. Dieser Artikel ist auf Springerlink.com mit Open Access verfügbar.

Zusammenfassung Dieser Beitrag betrachtet hochschulisches Lernen unter einer analytischen Perspektive: Danach artikuliert sich hochschulisches Lernen im institutionellen Kontext der Hochschule mit ihren studiengangstrukturellen Rahmenbedingungen in einer je spezifischen, dabei stets kontingenten und polymorphen Koppelung zwischen Wissenschaft als Ort der Wissensproduktion einerseits und Studium als Ort der Erschließung von Sinn- und Handlungsressourcen andererseits. Hochschulisches Lernen lässt sich als Möglichkeitsraum von Figurationen dieser Bezugnahmen rekonstruieren. Die Analyse des Lernens an Hochschulen erschließt außerdem Forschungsperspektiven einer bildungswissenschaftlichen Hochschulforschung und einer theoretischen Hochschuldidaktik, zu denen die Erwachsenenbildungswissenschaft substanzielle Beiträge liefern kann.

Schlüsselwörter Bildungswissenschaftliche Hochschulforschung ·

Hochschulisches Lernen · Studium

Abstract This article examines learning at the university, which is located in the institutional context of the university with its structural conditions. The analysis of learning at universities opens research perspectives, to which the adult education science can deliver substantial contributions.

Keywords education scientific research on higher education $\cdot$ learning at the university $\cdot$ academic studies

Dr. R. Rhein $(\bowtie)$

Leibniz Universität Hannover,

Hannover, Deutschland

E-Mail: ruediger-rhein@online.de 


\section{Hochschulisches Lernen - ein Gegenstand auch der Erwachsenenbildungswissenschaft}

Die Hochschullandschaft ${ }^{1}$ befindet sich im Wandel, die Studienstrukturreformen der letzten Jahre haben weitreichende Umstrukturierungen der Studienorganisation induziert, Durchlässigkeit des Hochschulzugangs und Anerkennung extern erworbener Kompetenzen sind Gegenstände aktueller Diskussion. ${ }^{2}$ Dies ist Anlass genug, nicht lediglich die Effekte dieser Entwicklungen durch forschende Begleitung präziser zu beschreiben, ,um auf diese Weise auch die unbeabsichtigten Nebenfolgen bildungspolitischer Entscheidungen genauer zu analysieren", 3 sondern auch grundlegend auf hochschulisches Lehren und Lernen zurückzudenken, um seinen konzeptuellen Kern theoretisch zu begründen. Die Erwachsenenbildungswissenschaft kann sich dabei als einschlägige Akteurin im transdisziplinären Feld bildungswissenschaftlicher Hochschulforschung verstehen. In diesem Sinne muss sich die Erwachsenenbildungswissenschaft nicht darauf beschränken, wissenschaftliche Weiterbildung ${ }^{4} \mathrm{zu}$ untersuchen; ebenso wenig braucht sie sich unter einheimischer Perspektive allenfalls für „öffentliche Wissenschaft“ zu interessieren, also für die „Frage nach der Vermittlung wissenschaftlichen Wissens an diejenigen, die nicht unmittelbar an der Wissenschaftsproduktion beteiligt sind“",5 oder für popularisierende Vermittlungsformen wissenschaftlichen Wissens. ${ }^{6}$ Die Erwachsenenbildungswissenschaft kann hochschulisches Lehren und Lernen in seiner Gesamtheit als genuin disziplinären Gegenstand auffassen - sehr wohl wissend, dass sie hier aus naheliegenden Gründen keine Alleinzuständigkeit reklamieren kann.

Dabei interessiert die Universität als Lernort in erwachsenenbildungswissenschaftlicher Hinsicht nicht lediglich deshalb, weil die Adressat/inn/en hochschulischer Bildung mündige Erwachsene sind. Vielmehr lassen sich Hochschulen (auch) als einschlägiger Bestandteil des Bildungssystems ${ }^{7}$ - insbesondere auch im Kontext des Lebenslangen Lernens ${ }^{8}$ - auffassen, um als Hochschule der Gesellschaft spezifische Lern- und Bildungsräume zu schaffen, in denen Bildungsbedürfnisse und Bildungsbedarfe individueller wie gesellschaftlicher Art befriedigt werden können.

Dabei bleibt natürlich weiterhin zu berücksichtigen, dass dem Lernort Hochschule eine nur transdisziplinär erfassbare Eigenlogik innewohnt:

Hochschulen im Allgemeinen, und Universitäten im Besonderen, sind konzeptuell $^{9}$ zunächst Trägerorganisationen von Wissenschaft und akademischen Praxen

\footnotetext{
${ }^{1}$ Vgl. exemplarisch Teichler und Tippelt 2005.

${ }^{2}$ Vgl. exemplarisch Faulstich 2011b.

${ }^{3}$ Friebertshäuser 2004, S. 248.

${ }^{4}$ Vgl. etwa die Schwerpunkthefte Report 31 (2008) 1 und Hessische Blätter für Volksbildung 62 (2012) 2. Vgl. exemplarisch auch Krüger 1984; Wittpoth 1987.

${ }^{5}$ Faulstich 2008, S. IV.

${ }^{6}$ Vgl. bspw. Conein et al. 2004.

${ }^{7}$ Vgl. exemplarisch Teichler 2009.

${ }^{8}$ Vgl. exemplarisch Wolter 2010; Kerres et al. 2012.

${ }^{9}$ Die Universität als „Idee“ ist zu unterscheiden von den empirisch vorfindlichen, durch lokale und historische Besonderheiten charakterisierten Hochschulen (vgl. zu diesem Gedanken Mecheril und Klingler 2010, S. 83 f.); außerdem differenziert sich die Idee der Universität noch einmal im Hinblick auf disziplinär
} 
sowie spezielle Orte des Umgangs mit spezifischen Formen des Wissens, des intellektuellen Austauschs und der intellektuellen Innovation. ${ }^{10}$

Zugleich aber sind Hochschulen nicht nur Forschungseinrichtungen, sie sind - als Organisation und als Institution ${ }^{11}$ - auch das Bindeglied zwischen Wissenschaft und Studium. ${ }^{12}$ Dieser Nexus erfordert somit eine Reflexion darüber, wie die Zwecke lernender Beschäftigung mit Wissenschaft mit Zwecksetzungen wissenschaftlicher Praxis synchronisiert und in typischen Auffassungen von der Institution Hochschule (bzw. einer Idee der Universität) artikuliert werden können.

Aus der Perspektive bildungswissenschaftlicher Hochschulforschung stellen sich dazu Fragen, die auch die Erwachsenenbildungswissenschaft als ihrige ansehen kann:

- Was genau bedeutet es, Wissenschaft zu einem Gegenstand von Lehre und Studium zu machen?

- Worin bestehen Ziel und Zweck der lernenden Auseinandersetzung mit Wissenschaft als Gegenstand des Studiums?

- Was zeichnet hochschulisches Lehren und Lernen aus?

- Was ist das Charakteristische akademischer Bildung?

Diese Fragen sollen hier aber nicht empirisch behandelt werden; vielmehr soll das Bedingungsgefüge ${ }^{13}$ von Hochschullehre, Studium und Wissenschaft systematisch expliziert werden, um genau diejenigen Aspekte zu identifizieren, die für hochschulisches, also auf den spezifischen Gegenstand Wissenschaft bezogenes Lehren und Lernen einschlägig sind.

\section{Die Eigenstrukturen von Wissenschaft}

Jede theoretische Rekonstruktion und jede empirische Beforschung von Lehre und Lernen an (wissenschaftlichen) Hochschulen muss zunächst den Eigen-Sinn und die Eigen-Logik von Wissenschaft veranschlagen:

bedingte Traditionen. Welcher Art die konzeptuellen Selbst- und Fremdbeschreibungen von Hochschulen im Allgemeinen bzw. von Universitäten im Besonderen tatsächlich sind, ist eine Frage der sozialwissenschaftlichen und historischen Hochschulforschung.

${ }^{10}$ Vgl. zur Universität allgemein Stichweh 1994; Weber 2002; Koch 2008.

${ }^{11}$ Institution verstanden als sozial entstandene und kollektiv geteilte, verfestigte handlungsleitende Sinnund Deutungszusammenhänge (vgl. Hillmann 2007, S. 381), Organisation verstanden als „ein soziales System oder ein soziales Gebilde als Gesamtheit aller geplanten, ungeplanten und unvorhergesehenen sozialen Prozesse, die innerhalb des jeweiligen Systems oder in Beziehung zu anderen, umgebenden Systemen ablaufen“ (Hillmann 2007, S. 651). Vgl. zum Verhältnis von Institution und Organisation Göhlich 2011.

${ }^{12}$ Vgl. exemplarisch Huber 1984; Teichler 2004.

${ }^{13}$ Dabei ist „Bedingung“ nicht in kausalem Sinne gemeint (etwas verursacht etwas, etwas hat etwas zur Folge), sondern in einem analytisch-deskriptiven Sinne - als Referenz auf Umstände oder Beziehungen, die gleichwohl nicht zufällig gegeben sind, sondern in gegenseitigen Verweisungszusammenhängen stehen und insofern im Hinblick auf eine inhärente Eigen-Logik rekonstruiert werden können. 
Lernen ist stets eine kognitiv-emotional-soziale Bezugnahme auf etwas - als Gegenstand des Lernens. ${ }^{14}$ Eine Untersuchung dieses Lernens muss auch die EigenStrukturen dieses „Etwas“ in Rechnung stellen, um beurteilen zu können, inwiefern das Lernen seinem Gegenstand gerecht wird oder diesen verfehlt, und um aufzuklären, inwiefern Sinn und Zweck des Lernens mit Sinn und Zweck des Lerngegenstandes korrespondieren.

Wissenschaft lässt sich explizieren als spezifische Praxis des Vernunftgebrauchs zu Erkenntniszwecken - also als Institution der methodisch ausgewiesenen und methodologisch begründeten Erzeugung von (gleichwohl potenziell vorläufigem) Wissen. ${ }^{15}$

Wissenschaft zeigt sich in der Pluralität der verschiedenen Disziplinen, die jeweils einschlägige Gegenstände und Fragestellungen als zur jeweiligen Fachwissenschaft zugehörig und jeweils spezifische Verfahren der Erkenntnisgewinnung als „wissenschaftlich" auszeichnen. Bei den Entitäten, über die Wissen erzeugt wird, handelt es sich, je nach Wissenschaftsdisziplin,

- um natürliche (extrapersonal-objektweltliche), um mentale und psychische (intrapersonale) oder um soziale, gesellschaftliche oder kulturelle (interpersonale bzw. trans- und suprapersonale) Sachverhalte, Phänomene und Prozesse (Realgegenstände),

- um(axiomatisierte)formale Systeme und ihre Eigenschaften(Formalgegenstände),

- um technische oder kulturelle Artefakte und die Bedingungen und Prozesse ihrer Erzeugung und Nutzung,

- um die Begründung und Gestaltung grundlegender menschlicher Praxen (der Sinndeutung, der Gestaltung gesellschaftlicher Verkehrsformen, der Gestaltung diskursiver Räume, der Gestaltung von Wertordnungen u. Ä.).

Neben Wissenschaft als epistemischem Projekt finden sich erstens unterschiedliche akademische Praxen als spezifisch wissensbasiertes und begründet-reflektiertes Handeln im Hinblick auf

- den sachkundigen oder verantwortlichen Umgang mit Realgegenständen,

\footnotetext{
${ }^{14}$ Damit ist natürlich keine Definition von Lernen gegeben! Mit dem Ziel einer explizit pädagogischen Erfassung des Phänomens „Lernen“ formulieren Göhlich und Zirfas (2007, S. 17) folgenden „Arbeitsbegriff“: „Lernen bezeichnet die Veränderungen von Selbst- und Weltverhältnissen sowie von Verhältnissen zu anderen, die nicht aufgrund von angeborenen Dispositionen, sondern aufgrund von zumindest basal reflektierten Erfahrungen erfolgen und die als dementsprechend begründbare Veränderungen von Handlungs- und Verhaltensmöglichkeiten, von Deutungs- und Interpretationsmustern und von Geschmacksund Wertstrukturen vom Lernenden in seiner leiblichen Gesamtheit erlebbar sind; kurz gesagt: Lernen ist die erfahrungsreflexive, auf den Lernenden sich auswirkende Gewinnung von spezifischem Wissen und Können.“

${ }^{15}$ Zur Unterscheidung von anderen Wissensformen werden für wissenschaftliches Wissen spezifische Kriterien reklamiert. Hoyningen-Huene (2009, S. 22) identifiziert Systematizität ,als das, was Wissenschaft ausmacht": „Wissenschaftliches Wissen unterscheidet sich von anderen Wissensarten, besonders dem Alltagswissen, primär durch seinen höheren Grad an Systematizität", wobei wissenschaftliches Wissen in neun Dimensionen systematischer ist als andere Wissensarten, nämlich hinsichtlich 1) Beschreibungen, 2) Erklärungen, 3) Vorhersagen, 4) der Verteidigung von Wissensansprüchen, 5) kritischem Diskurs, 6) epistemischer Vernetztheit, 7) dem Ideal der Vollständigkeit, 8) der Vermehrung von Wissen und 9) der Strukturierung und Darstellung von Wissen - ohne dass alle neun Dimensionen für alle Wissenschaften zwingend einschlägig sind (vgl. ausführlich Hoyningen-Huene 2013). Unbenommen bleibt, dass von „der“ Wissenschaft im Kollektivsingular nur in allgemeiner Hinsicht gesprochen werden kann.
} 
- den sachkundigen Entwurf technischer oder kultureller Artefakte, ihre sachverständige Produktion oder den adäquaten Umgang mit diesen,

- sozial-kommunikative Prozesse in grundlegenden lebensweltlichen Praxen.

Und zweitens findet sich die reflektiert-routinierte Verwendung gesicherter Wissensbestände in Form von Technologien. ${ }^{16}$

Akademische Praxen verwenden zum Zwecke solches verantwortlichen und sachkundigen Verrichtens, gekonnten Entwerfens, Konstruierens und Gestaltens oder professionellen Kommunizierens, Handelns und Urteilens nicht nur theoretisches, sondern auch praktisches, technisches und reflektorisches Wissen, das aus überindividuell verfügbaren, aktual bewährten Erfahrungen oder aus diskursiv-reflexiven Einsichten gewonnen wurde.

Die einzelnen Fächer sind typische integrale Verkoppelungen von wissenschaftlichen Disziplinen und akademischen Praxen: Hier werden analytisch-empirische, theoretische, konzeptuell-programmatische, ethische, praxeologisch-operative sowie reflexive Umgangsmodalitäten mit den einschlägigen Gegenständen und Problemhorizonten auf eine je typische Weise konfiguriert (vgl. Abb. 1). Unbenommen bleibt, dass viele Fächer eine ausschließlich wissenschaftliche Ausrichtung aufweisen können. Andere Fächer dagegen greifen konstitutiv auch auf akademische Praxen zurück, wenn sie mit ihren Gegenständen nicht nur theoretisch, beobachtungswissenschaftlich oder hermeneutisch umgehen, sondern auch ein verantwortliches Handeln bzw. ein gekonntes Verrichten, Gestalten oder Intervenieren beabsichtigen (dies gilt beispielsweise für die Fachfamilien der Ingenieurwissenschaften, Medizin oder Rechtswissenschaft) diese Fächer sind insofern auf Wissenschaft bezogen, als sie eine epistemisch-theoretische Basis haben, sie gehen aber über ein eng gefasstes epistemisches Verständnis von Wissenschaftlichkeit immer dann hinaus, wenn sie neben theoretischer Erkenntnis-, Urteils- und Reflexionsfähigkeit auch aisthetische, diagnostische, normativ-ethische oder praktische Urteilsfähigkeit oder poietische Gestaltungsfähigkeit einfordern.

Bedeutsam für die Analyse akademischen Lernens ist das Zurückdenken auf Ziel und Zweck der jeweiligen Fächer - und auf Ziel und Zweck des Studiums dieser Fächer.

Das Ziel wissenschaftlicher Tätigkeit ist die Produktion wissenschaftlichen Wissens und der Ausweis seiner Geltungsbedingungen - Wissenschaft ist ein epistemisches Projekt.

Davon zu unterscheiden sind die Zwecke wissenschaftlicher Praxis, die mit dem Betreiben wissenschaftlicher Fächer verfolgt werden, wobei nochmals zu unterscheiden ist zwischen 1) der Frage, wozu Wissenschaft betrieben bzw. wozu wissenschaft-

\footnotetext{
${ }^{16}$ Stichweh (2006) stellt heraus, dass ,nahezu alle an der Universität vorhandenen Disziplinen nicht nur Wissen produzieren und vermitteln, sondern Teilbeständen dieses Wissens die Form von Technologien geben“ (Stichweh 2006, S. 45). „Für ein solches Verständnis benötigt man einen allgemeineren Begriff von Technologie, den man im Anschluss an Luhmann so formulieren kann, dass von Technologie dort die Rede ist, wo ein Verfahren oder eine Handlungsweise wiederholt und von verschiedenen Personen benutzt werden kann, ohne dass die Nutzer einer Technologie alle Sinnimplikationen, die der Technologie zugrunde liegen, nachzuvollziehen gezwungen sind (vgl. Luhmann 1975, S. 70 ff.). Dieser Verzicht auf Nachvollzug und Prüfung aller zugrundeliegenden Sinnimplikationen macht gerade die handlungspraktische Ökonomie von Technologien aus und wird zur Bedingung ihrer sozialen Universalisierbarkeit" (Stichweh 2006, S. 43).
} 
Abb. 1 Fächer als typische integrale Verkoppelungen von wissenschaftlichen Disziplinen und akademischen Praxen

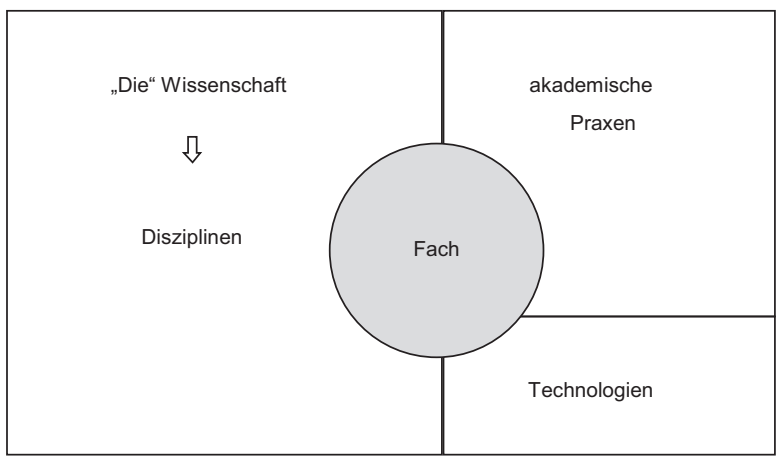

liches Wissen gewonnen wird, also der Frage nach den Erkenntnisinteressen, und 2) den Praxen der Verwendung bzw. der Transformation wissenschaftlichen Wissens und den inner- und außerwissenschaftlichen Anschlussoptionen wissenschaftlicher Fächer und wissenschaftlich orientierter Tätigkeit.

Hinsichtlich der Erkenntnisinteressen ${ }^{17}$ lassen sich mindestens folgende Zweckbestimmungen rekonstruieren:

- Befriedigung epistemischer Interessen und theoretischer Neugierde - als Erzeugung theoretischen Wissens in Form von systematischen Beschreibungen, Erklärungen oder Verstehenshorizonten und Ausweis ihrer Geltungsbedingungen;

- Befriedigung poietischer (d. h. auch: technologischer) Interessen - als Erzeugung von Verfügungswissen über Ressourcen und Prozesse im Hinblick auf die geschickte Produktion von Artefakten oder auf zielführendes, effizientes Verrichten;

- Befriedigung praktischer Interessen - als Erzeugung von Reflexionswissen über Formen und Ermöglichungsbedingungen individueller bzw. kollektiver leiblichseelischer, ökologischer, ökonomischer, kultureller oder ethischer lokaler bzw. globaler lebenspraktischer Grundlagen und gelingender Lebensvollzüge und den Theoriefiguren zu ihrer Rechtfertigung.

Diese Zwecksetzungen können durchaus wechselseitig aufeinander bezogen sein, weil beispielsweise technisch-verdinglichte Verfügungen lebenspraktische Grundlagen mitgestalten und umgekehrt die Gestaltung lebenspraktischer Grundlagen auch technisch-verdinglichter Voraussetzungen bedarf; beides ruht auf epistemischen Konstellationen, und diese induzieren ihrerseits technologische und praktisch-lebensweltliche Verwendungsmöglichkeiten.

Neben den Erkenntnisinteressen als Zwecksetzungen von Wissenschaft lassen sich idealtypisch verschiedene inner- und außerwissenschaftliche Anschlussoptionen

\footnotetext{
${ }^{17}$ Diese Skizzierung der Ziele wissenschaftlicher Tätigkeit ist ein heuristisch gemeinter Vorschlag zur Rekonstruktion der Bildungsziele der Hochschule, über die sich Hochschullehre Klarheit verschaffen muss. Mein Vorschlag greift - ohne dieses im Detail auszuweisen und ohne es explizit zu begründen - auf die habermassche Trias von technischem, praktischem und emanzipatorischem Erkenntnisinteresse (vgl. Habermas 1968) sowie auf die aristotelische Trias von theoria, poiesis und praxis zurück.
} 
an wissenschaftliche Tätigkeit (bzw. an akademische Fächer) und Praxen der Verwendung bzw. der Transformation wissenschaftlichen Wissens unterscheiden: ${ }^{18}$

- Wissenschaft selbst - als Bearbeitung selbstreferenziell generierter Forschungsfragen mit dem Ziel der Erweiterung des Wissens - unbeschadet der Möglichkeit einer späteren Verwendung der Erkenntnisse für andere Zwecke (innerwissenschaftliche Anschlussoption;

- wissenschaftliche Expertise - als Bearbeitung von singulären und kontextuell verorteten Fragen, deren Beantwortung zwar wissenschaftliche Methoden erfordert, aber keinen allgemeinen wissenschaftlichen Erkenntnisgewinn erzielt, sondern lediglich einen konkreten Sachverhalt aufklärt (außerwissenschaftliche Anschlussoption, bei der Angehörige des Wissenschaftssystems als wissenschaftliche Experten mit Angehörigen anderer gesellschaftlicher Subsysteme interagieren);

- Transdisziplinarität - als Vermittlung zwischen Wissenschaft und außerwissenschaftlichen Anliegen (außerwissenschaftliche Anschlussoption, bei der im Unterschied zur Expertise das wissenschaftliche Wissen nicht exklusiv bei wissenschaftlichen Experten verbleibt, sondern geteilt wird, um durch das Teilen des Wissens zu neuen Einsichten oder Einschätzungen zu kommen);

- akademische Professionalität - als die sachkundige und sachverständige Bearbeitung komplexer Fragen und Problemstellungen in akademisierten Tätigkeitsfeldern und als Prozess der Generierung von Lösungen, dem die Entwicklung und Prüfung von Wissen nicht nur vorausgeht, sondern inhäriert (außerwissenschaftliche Anschlussoptionen, wobei wissenschaftliche Erkenntnisse oder wissenschaftliche Methoden verwendet werden, ohne dass hier der Anspruch erhoben würde, Wissenschaft zu betreiben).

\section{Wissenschaft als Lerngegenstand - Anforderungshorizonte im Studium}

Das Ziel des Studiums ist idealerweise ${ }^{19}$ eine reflektierte Auseinandersetzung mit dem jeweiligen Fach und seinen spezifischen Formen der Erschließung von Sachverhalten.

Studieren bedeutet, die Eigenstrukturen akademischer Disziplinen hinsichtlich ihrer epistemischen, poietischen und praktischen Potenziale verstehend anzueignen und praxeologisch zu erschließen. ${ }^{20}$

Die Eigenstrukturen ergeben sich aus der Tatsache, dass den jeweiligen Fächern spezifische Grundideen der Gegenstandskonstitution und der Gegenstandsbehandlung zugrunde liegen; die Fächer pflegen je spezifische Herangehensweisen an Pro-

\footnotetext{
${ }^{18}$ Dabei knüpfen die innerwissenschaftlichen Anschlussoptionen unmittelbar an das theoretische Erkenntnisinteresse an, während sich die außerwissenschaftlichen Anschlussoptionen in den Dienst sowohl poietischer als auch praktischer Interessen stellen können.

${ }^{19}$ Die Rede von einem Ideal stellt die Möglichkeit in Rechnung, dass sich die Sachverhalte faktisch anders darstellen. Aussagen über Ideales gehören in einen explikativen Diskurs, Aussagen über Faktisches in einen deskriptiven, empirisch fundierten Diskurs. Vgl. zu diesen Diskursebenen Schnädelbach 1977.

${ }^{20}$ Diese Aussage ist normativ insofern, als hier ein Bedeutungshorizont (re-)konstruiert und somit als Maßstab konstituiert wird.
} 
blemlösungen und Bearbeitungsmodi von Aufgabenstellungen und haben hierfür typische, disziplinbezogene Denkweisen, Konzepte, Methoden und Techniken entwickelt. Somit stellt sich für jede Wissenschaftsdisziplin die Frage: Auf welche Art und Weise werden die relevanten Realitätsausschnitte in Theorien, Modellen oder anderen Arten von Rekonstruktionen fasslich und (vor allem für das Denken) operabel gemacht, um letztlich die betreffenden Sachverhalte der Wirklichkeit zu verstehen, zu erklären, vorherzusagen, praktisch zu nutzen und zu ändern? ${ }^{21}$

Studieren als verstehendes Aneignen des Eigen-Sinns von Wissenschaft bedeutet, zu begreifen, auf welche Fragen und Problemstellungen Wissenschaft jeweils eine Antwort geben will. Es geht dabei nicht nur um Verständlichkeit (im kognitionspsychologischen Sinn), sondern insbesondere um Verstehbarkeit (im hermeneutischen Sinn). Gemeint ist hier die Replikstruktur der Hermeneutik: „Man versteht etwas, indem man es versteht als Antwort auf eine Frage; anders gesagt: man versteht es nicht, wenn man nicht die Frage kennt und versteht, auf die es die Antwort war oder ist." 22

Studieren als verstehendes Aneignen des Eigen-Sinns von Wissenschaft bedeutet, im Lerngegenstand die subjektive Bedeutsamkeit zu entdecken - ohne dabei die objektiven Anforderungshorizonte, die durch den Gegenstand gesetzt sind, zu unterlaufen, und sich diese objektiven Anforderungshorizonte als subjektiv bedeutsam zu erschließen. Für jedes sinnhafte Lernen dürfte gelten: „Man lernt nicht nur etwas, indem man es rezeptiv aufnimmt, sondern indem man das Fremde mit dem Eigenen verknüpft." ${ }^{23}$ Solches Lernen , ist gekennzeichnet durch einen bipolaren Prozess, der durch das Eindringen der Außenwelt in den Lernenden und das Eindringen des Lernenden in die Außenwelt verstanden werden muss. "24

Verstehendes Aneignen mündet darin, nicht nur Antworten und die zugrunde liegenden Fragen zu verstehen, sondern auch, sich wissenschaftliche Frage- und Problemstellungen zu eigen zu machen und schließlich selbst sinnvolle Fragen zu stellen, hierauf Antworten zu suchen und eigene Erkenntnisinteressen zu artikulieren.

Sich die epistemischen Potenziale von Wissenschaft - als einer Praxis des Erklärens und Verstehens - zu erschließen, bedeutet, das wissenschaftliche Erklären und Verstehen selbst zu verstehen. Studieren bedeutet nicht, Wissenschaft als System abstrakter Aussagen zu erlernen. Vielmehr muss, wer studiert, verstehen, wie Wissenschaft zu ihren Erkenntnissen kommt, und wie sie den Anspruch auf Geltung dieser Erkenntnisse begründet. Studieren bedeutet somit, den spezifischen Charakter wissenschaftlichen Wissens zu begreifen, das heißt u. a.

- die Funktion von Theorie und theoretischer Abstraktion zu verstehen;

- das Verhältnis von Theorie und Poiesis/Praxis zu begreifen, also die Zwecke wissenschaftlichen Tuns und die Verwendungsoptionen von Wissenschaft zu verstehen;

\footnotetext{
${ }^{21}$ Vgl. Heckhausen 1987, S. 132.

${ }^{22}$ Marquard 1981, S. 118 mit Bezug auf Gadamer, Wahrheit und Methode, 1960.

${ }^{23}$ Göhlich und Zirfas 2007, S. 37.

${ }^{24}$ Göhlich und Zirfas 2007, S. 56.
} 
- zu wissen, dass - zumindest empirisches - wissenschaftliches Wissen häufig abduktiv gewonnen wird;

- zu wissen, dass wissenschaftliches Wissen in der Regel paradigmatisch situiert ist;

- zu wissen, dass wissenschaftliches Wissen üblicherweise spezifischen Diskursarenen mit jeweils eigenen Begrifflichkeiten und Gegenstandsprämierungen entstammt;

- zu durchschauen, welche potenziellen Verkürzungen und Vereinseitigungen das disziplinäre Wissen enthält.

Das Ziel bei der Erschließung der epistemischen Potenziale der Disziplin ist also, zu begreifen, welche Erkenntnisse sich mit den je besonderen Methoden der jeweiligen Wissenschaft gewinnen lassen, und die disziplinbezogenen Fähigkeiten zur Wahrnehmung, zum Erkennen, zum Methodengebrauch, zum Urteilen und zum Reflektieren zu entwickeln (vgl. Abb. 2).

Sich die poietischen Potenziale von Wissenschaft zu erschließen bedeutet, Formen der Nutzbarmachung wissenschaftlichen Wissens zu erschließen (und dies nicht notwendigerweise nur in technologischer Hinsicht, sondern auch als Potenzial im Hinblick auf die Bearbeitung von Schlüsselproblemen).

Sich die praktischen Potenziale von Wissenschaft $\mathrm{zu}$ erschließen bedeutet, die Sinndimension wissenschaftlichen Wissens zu bearbeiten, denn wissenschaftliche Erkenntnisse haben Aus- und Rückwirkungen auf das menschliche Selbstverständnis. Hier ist nicht nur zu fragen, welcher Sinn wissenschaftlichen Erkenntnissen abgewonnen werden kann, sondern auch, welche Interpretationsoptionen für das menschliche Selbstverständnis im Lichte wissenschaftlicher Erkenntnisse bestehen und wie diese (kritisch) zu bewerten sind:

Wissenschaft ist zwar immer bezogen auf ihren je konkreten Erkenntnisgegenstand. Zugleich ist das erkennende Subjekt aber immer auch Teil derjenigen Welt, über die es aus der Position eines distanzierten wissenschaftlichen Beobachters etwas erkennen will; es besteht offenbar ein „dialektische[s] Verhältnis von Zugehörigkeit

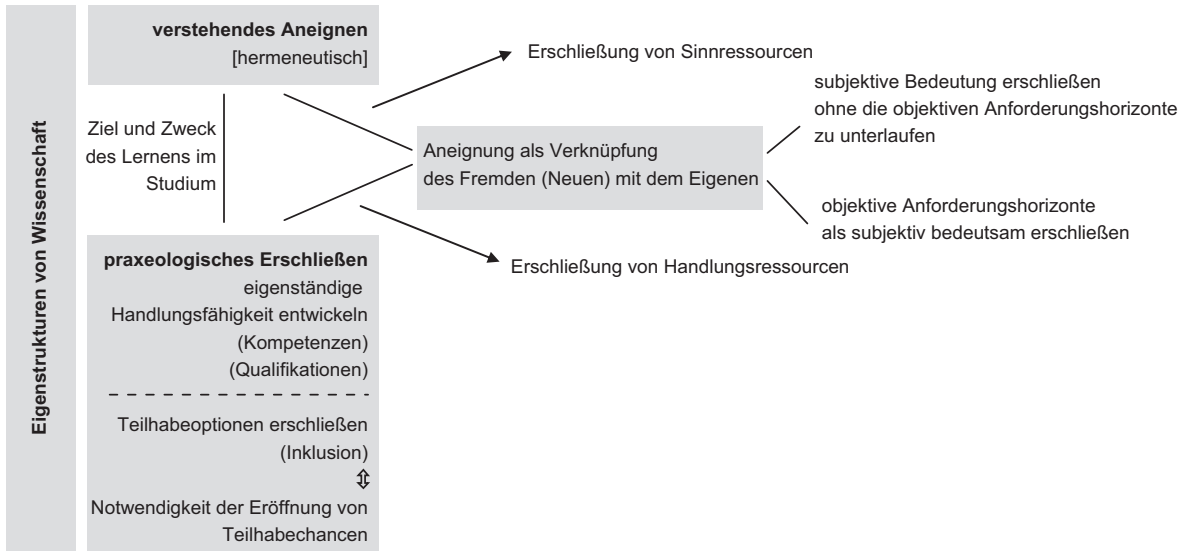

Abb. 2 Studium als Erschließung von Sinn- und Handlungsressourcen 
und Distanzierung, das unsere Stellung in der Welt charakterisiert" ${ }^{25}$ Weil das erkennende Subjekt also unhintergehbar Teil derjenigen Welt ist, über die es aus der Position distanzierter wissenschaftlicher Beobachtung etwas erkennen will, haben seine Erkenntnisse über diese Welt, über Natur, Mentales oder Soziales stets auch Auswirkungen auf sein Selbstverständnis.

Studieren als praxeologisches Erschließen der Potenziale von Wissenschaft bedeutet, fachbezogene Praxen der Verwendung bzw. der Transformation wissenschaftlichen Wissens kennenzulernen, in diesen innerwissenschaftlichen und fachbezogen-außerwissenschaftlichen Praxen Erfahrungen $\mathrm{zu}$ sammeln und diese Erfahrungen zu reflektieren, um auf diese Weise ein spezifisches Wissen und Können aufzubauen.

Das Ziel des Studiums ist, eine eigenständige Handlungsfähigkeit zu entwickeln, und selbst gemäß fachlich-disziplinärer Sinnstrukturen tätig werden zu können.

Studieren bedeutet dann, sich Teilhabeoptionen an inner- oder außerwissenschaftlichen Praxen zu erarbeiten.

\section{Das Studium als performative Bezugnahme auf Wissenschaft und als Erschließung von Sinn- und Handlungsressourcen}

Als Lerngegenstand fordert Wissenschaft, mit ihren Eigenstrukturen auf angemessene Weise umzugehen: Die Studierenden sehen sich bei ihren performativen Bezugnahmen auf Wissenschaft mit bestimmten Anforderungen konfrontiert, die sich als Gelingensbedingungen eines Hochschulstudiums lesen lassen.

Hochschulisches Lernen artikuliert sich dann in einer je spezifischen, dabei stets kontingenten und polymorphen Koppelung zwischen Wissenschaft als besonderer Praxis einerseits und Studium als performativer Bezugnahme auf diese Praxis andererseits. Dabei ist in Rechnung zu stellen, dass empirisch beobachtbares studierendes Lernen seinen Gegenstand auf unterschiedliche Weise verfehlen kann.

Studierende sind dabei nicht lediglich epistemische Subjekte, die sich akademischem Lernen unterziehen, um sich anschließend am wissenschaftlichen Diskurs um der Wahrheitssuche willen beteiligen. Sie sind auch psycho-physische Subjekte, die im Zuge ihrer Lernprozesse kognitive Operationen, spezifische Motivlagen und emotionale Valenzen selbstgesteuert regulieren müssen und ihren Lernhandlungen einen Eigen-Sinn verleihen, sie sind psycho-soziale Subjekte, die sich im Hinblick auf Sinn- und Handlungsressourcen Teilhabeoptionen verschiedenster Art zu erarbeiten versuchen, und sie sind sozial-kulturelle Subjekte, deren lernende Auseinandersetzung mit Wissenschaft in biografische und spezifisch lebensweltlich kontextualisierte Aneignungssituationen eingebettet ist.

Die lernende Auseinandersetzung mit Wissenschaft erfordert die Erschließung von Sinn- und Handlungsressourcen. Für die Studierenden bedeutet dies, die Frage zu klären, welche spezifische (subjektive) Bedeutsamkeit die Beschäftigung mit dem eigenen Studienfach jeweils hat, und welche spezifischen Zwecksetzungen sich mit dem Studienfach verfolgen lassen.

\footnotetext{
${ }^{25}$ Mattern 1996 zum Kerngedanken der hermeneutischen Philosophie Paul Ricœurs.
} 
Eine originäre Sinnressource mag darin bestehen, an persönliche Interessen, Begabungen oder Motivlagen anzuknüpfen, die sich durch die Beschäftigung mit dem Studienfach mutmaßlich befriedigen lassen. Eine spezifische Transformationsleistung im Verlauf des Studiums besteht dann aber darin, an Erkenntnisinteressen anzuschließen, die durch das jeweilige Fach begründet werden können.

Originäre Handlungsressourcen mögen darin bestehen, Bildungszertifikate zu erwerben oder eine Lizenz, Approbation oder Zulassung für ein bestimmtes Tätigkeitsfeld zu erhalten. Eine spezifische Transformationsleistung im Verlauf des Studiums besteht dann aber darin, an die inner- und außerwissenschaftlichen Anschlussoptionen des jeweiligen Faches anknüpfen: Der Zweck eines Studiums kann ja darin bestehen, 1) sich auf ein bestimmtes (professionelles) Tätigkeitsfeld vorzubereiten, das die Nutzung (fach)wissenschaftlichen Wissens und (fach)wissenschaftlicher Methoden bzw. Technologien zur Lösung oder Bearbeitung spezifischer Problemstellungen verlangt (also Verfügungswissen über Ressourcen und Prozesse zu erwerben), oder 2) Wissenschaftler/in zu werden (und dafür neben dem wissenschaftlichem Wissen fundierte methodische und methodologische Kenntnisse zu erwerben, um eigene Fragestellungen forschend zu bearbeiten), oder 3) eine spezifisch intellektuelle Haltung einzuüben, mit dem Ziel, komplexe Sachverhalte aufzuklären, zu dechiffrieren und zu verstehen und zwischen Wissenschaft und außerwissenschaftlichen Anliegen zu vermitteln.

\section{Studieren und Lernen - eine analytische Matrix}

Vor dem Hintergrund der Explikation des Bedingungsgefüges von Hochschullehre, Studium und Wissenschaft lassen sich jetzt (im Sinne stipulativer Definitionen ${ }^{26}$ ) begriffliche Präzisierungen nachtragen.

Der Begriff „akademisch“ artikuliere eine einschlägige Steigerung des zentralen Kriteriums desjenigen Modus, in dem auf den Gegenstand der jeweiligen Praxis spezifisch Bezug genommen wird.

Anders ausgedrückt: Menschliche Praxen lassen sich rekonstruieren als Befassung mit jeweils spezifischen Entitäten (Gegenständen, Sachverhalten usw.) in einem für die jeweilige Praxis einschlägigen Modus. Hier soll nun unterstellt werden, dass dieser Modus durch ein zentrales Kriterium charakterisierbar ist, das zugleich einschlägige Steigerungen erfahren kann. Der Begriff ,,akademisch“ artikuliere dann die höchste Steigerung dieses Kriteriums hinsichtlich bestimmter Parameter. ${ }^{27}$

\footnotetext{
${ }^{26}$ Die folgenden Unterscheidungen verstehen sich als begriffliche Unterscheidungen. In anderem Zusammenhang mögen sich hinter diesen Wortmarken andere Begriffsverständnisse verbergen; dies ändert aber nichts an der grundsätzlichen konzeptuellen Unterscheidung - es wären dann lediglich die hier verwendeten Wortmarken durch andere zu ersetzen.

${ }^{27}$ Im Hinblick auf Wissenschaft bedeutet dies: Wissenschaft als Praxis nimmt auf ihre [Erkenntnis]Gegenstände epistemisch Bezug; dieser epistemische Modus ist durch das zentrale Kriterium der Systematizität charakterisiert, wobei wissenschaftliches Wissen (nach Hoyningen-Huene 2009) im Hinblick auf 9 Dimensionen (Parameter) systematischer (!) ist als andere Wissensarten. Welches zentrale Kriterium nun für andere Modi (künstlerisch, theologisch, pädagogisch usw.) zu veranschlagen ist, um diese unter das Prädikat ,akademisch“ zu subsumieren, kann an dieser Stelle nicht erörtert werden.
} 
Der Begriff ,„akademisch“ ist somit ein Oberbegriff für spezifische Auszeichnungen menschlicher Praxen. Unter diesen Begriff fällt dann insbesondere (und dominant, aber nicht exklusiv) das Prädikat „wissenschaftlich“.

Der Oberbegriff „Hochschule“ referiere auf diejenigen Organisationen, in denen eine akademische Befassung mit Gegenständen, Sachverhalten usw. erfolgt, d. h. eine Befassung, die in Bezug auf die zentralen Kriterien des bezugnehmenden Modus spezifisch gesteigert ist.

Formal referiert der Begriff „Hochschule“ auf Universitäten, Fachhochschulen und sonstige Hochschulen (Kunsthochschulen, theologische Hochschulen usw.).

In den bisherigen Ausführungen wurde wesentlich auf wissenschaftliche Hochschulen und Universitäten fokussiert.

Hochschullehre ${ }^{28}$ ist eine spezifische Praxis themenfokussierter Adressierung von Studierenden im Modus einer spezifischen Bezugnahme auf Wissenschaft bzw. auf ihre Anschluss- und Verwendungsoptionen.

Studieren ist die lernende und zugleich performative Bezugnahme auf Wissenschaft bzw. akademische Praxen zu spezifischen Zwecken.

Studieren artikuliert sich in verschiedenen Formen und lässt sich in verschiedenen Formatierungen rekonstruieren.

Artikulationsformen des Studierens sind:

- Lernen im Studium (studentisches Lernen) ${ }^{29}$ - als sämtliche Lernprozesse im Verlauf des Studiums (als Topografie von Lernorten), unabhängig davon, ob sich diese Settings unmittelbar auf Wissenschaft beziehen oder nicht, und ob sie direkt an Hochschulen (als Organisationen) angebunden sind oder nicht. ${ }^{30}$

- Hochschulisches Lernen - als Prozesse und Ergebnisse desjenigen Lernens, das an Hochschulen (als Institutionen) stattfindet, unabhängig davon, welchen genauen Stellenwert dabei Wissenschaft einnimmt. ${ }^{31}$

- Akademisches Lernen - als Prozesse und Ergebnisse desjenigen Lernens, das Wissenschaft (bzw. akademische Praxen) zum Gegenstand hat, unabhängig vom spezifischen Lernort (der gleichwohl üblicherweise auf die Hochschule, als Institution bezogen sein wird ${ }^{32}$ ).

\footnotetext{
${ }^{28}$ Lehre allgemein ist eine auf spezifische Weise Handlungsintentionen entwerfende und begründende Haltung der Vermittlungsabsicht gegenüber Adressaten, damit als strukturtheoretisch explizierbare Position in einem mit charakteristischem Eigen-Sinn ausgestatteten Interaktionskontext (Lehr-Lern-Situation), in dem der Umgang mit typisch beschränkten Gelingensbedingungen, transintentionalen Handlungsfolgen und potenzieller Störanfälligkeit des Beziehungsgeschehens ausdrücklich veranschlagt wird. Vgl. zu Lehre allgemein Helsper 2011, fokussiert auf Hochschule Pazzini et al. 2010.

${ }^{29}$ Dieses Lernen kann im Kontext eines grundständigen Studiums oder im Kontext eines Weiterbildungsstudiums verschiedenster Art erfolgen.

${ }^{30} \mathrm{Im}$ Gegensatz zu den anderen Artikulationsformen finden sich hier beispielsweise Lernprozesse in außerhochschulischen, aber studiengangbezogenen Praktika, oder Lernprozesse im Zuge studentischer Selbstverwaltung.

${ }^{31} \mathrm{Im}$ Gegensatz zu den anderen Artikulationsformen finden sich hier beispielsweise der Erwerb von Fachsprachen oder von Schlüsselkompetenzen, oder auch Lernprozesse im Zuge der psycho-physischen oder psycho-sozialen Verarbeitung oder Reflexion von kognitiven, sozialen oder emotionalen Anforderungen des Studiums und Lernprozesse zur Entwicklung fachbezogener Identität oder zur (reflexiven) Vorbereitung auf fachbezogene Tätigkeitsstrukturen.

${ }^{32}$ In diesem Falle ist akademisches Lernen dann eine echte Teilmenge des hochschulischen Lernens. Akademisches Lernen außerhalb der Hochschule wäre zudem allenfalls denkbar im Kontext außerhochschulischer Forschungseinrichtungen.
} 
- Studierendes Lernen - als Integral sämtlichen Lernens im Kontext von Hochschule, Wissenschaft und Studium als biografisch verortetem Lernprojekt. ${ }^{33}$

Das Studium ist formatierbar als

- Topografie von Lernorten und Lernsettings, die geprägt sind durch die Institution Wissenschaft, die Organisation Hochschule und Ansprüche relevanter Umwelten der Hochschulen;

- Ort der Erschließung von Sinn- und Handlungsressourcen durch performative Bezugnahme auf Wissenschaft und akademische Praxen;

- Prozess einer lernenden Auseinandersetzung mit Wissenschaft und ihrem Eigen-Sinn;

- biografischer Abschnitt, der den Studierenden bestimmte fachliche, lebensweltliche und entwicklungsbedingte kognitiv-sozial-emotionale Leistungen abverlangt und der sich durch verschiedene explizite und implizite Lernmotive auszeichnet.

Der heuristische Wert dieser Unterscheidungen stellt sich dann ein, wenn von einer losen Koppelung dieser Artikulationsformen des Studierens mit rekonstruierbaren Formatierungen des Studiums ausgegangen wird (vgl. Tab. 1).

\section{Ausblick: Perspektiven bildungswissenschaftlicher Hochschulforschung}

Hochschulisches Lehren und Lernen lässt sich als zentraler Gegenstand bildungswissenschaftlicher Hochschulforschung auffassen.

Bildungswissenschaftliche Hochschulforschung kann mindestens drei Perspektiven betonen:

1. Bildungstheoretische Hochschulforschung - mit dem Leitmotiv, die Bedeutung der „Beschäftigung mit Wissenschaft“ analytisch aufzuklären.

2. (Rekonstruktive) bildungswissenschaftliche Hochschulforschung im engeren, empirischen Sinne - mit dem Leitmotiv, Lehren und Lernen an Hochschulen systematisch empirisch zu erschließen.

3. Kritik der Hochschuldidaktik zum Zwecke ihrer theoretischen Grundlegung, und daran anschließend theoretische Hochschuldidaktik zum praktischen Zwecke mit dem Leitmotiv, die Komplexität der Hochschullehre nicht vorschnell zu reduzieren, sondern auszuarbeiten und der Reflexion zugänglich zu machen.

Die vorangegangenen Ausführungen dienten einer analytischen Explikattion des Lernens an (wissenschaftlichen) Hochschulen.

Dieses Lernen ist geprägt durch

\footnotetext{
${ }^{33}$ Veranschlagt wird also die Möglichkeit (und die Sinnhaftigkeit) einer begrifflichen (!) Unterscheidung zwischen studierendem und studentischem Lernen: Während studentisches Lernen (= Lernen im Studium) unter einer objektiv-bilanzierenden Perspektive erscheint, liegt studierendem Lernen das Deutungsmuster einer emphatischen und somit subjektiv-bedeutsamen Bezugnahme auf den Lerngegenstand zugrunde, die performativ auf spezifische Weise lernend, nämlich studierend i. S. v. neugierig erkundend erfolgt.
} 
Tab. 1 Formatierungen des Studiums und Artikulationsformen des Studierens

\begin{tabular}{|c|c|c|c|}
\hline $\begin{array}{l}\text { Formatierung des Studiums } \\
\text { als... }\end{array}$ & $\begin{array}{l}\text { Artikulations- } \\
\text { formen des } \\
\text { Studierens }\end{array}$ & Bezugspunkt & Zentrale Aspekte \\
\hline $\begin{array}{l}\text { Topografie von Lernorten } \\
\text { und Lernsettings }\end{array}$ & $\begin{array}{l}\text { Lernen im } \\
\text { Studium }\end{array}$ & $\begin{array}{l}\text { Hochschule als } \\
\text { Organisation, Tätig- } \\
\text { keitsstrukturen und } \\
\text { Arbeitsfelder als } \\
\text { relevante Umwelten } \\
\text { der Hochschule }\end{array}$ & $\begin{array}{l}\text { Strukturlogik fremd- und } \\
\text { selbstarrangierter Settings } \\
\text { formellen und informellen } \\
\text { Lernens, das „Arrangement“ } \\
\text { als Ausdruck ,pädagogischer } \\
\text { Form“ }\end{array}$ \\
\hline $\begin{array}{l}\text { Ort der Erschlie- } \\
\text { ßung von Sinn- und } \\
\text { Handlungsressourcen }\end{array}$ & $\begin{array}{l}\text { hochschulisches } \\
\text { Lernen }\end{array}$ & $\begin{array}{l}\text { Hochschule als } \\
\text { Institution }\end{array}$ & $\begin{array}{l}\text { Fachkulturen, } \\
\text { Hochschulsozialisation }\end{array}$ \\
\hline $\begin{array}{l}\text { lernende Auseinandersetzung } \\
\text { mit Wissenschaft und ihrem } \\
\text { Eigen-Sinn }\end{array}$ & $\begin{array}{l}\text { akademisches } \\
\text { Lernen }\end{array}$ & $\begin{array}{l}\text { Wissenschaft und } \\
\text { akademische Praxen } \\
\text { als Institutionen }\end{array}$ & $\begin{array}{l}\text { Wissenschaftsreflexion, } \\
\text { Lernen als kognitiv-sozial- } \\
\text { emotionale Bezugnahme } \\
\text { auf etwas (als Lerngegen- } \\
\text { stand), Sinnrekonstruktion } \\
\text { als realstrukturtheoretische } \\
\text { Hermeneutik }\end{array}$ \\
\hline biografischer Abschnitt & $\begin{array}{l}\text { studierendes } \\
\text { Lernen }\end{array}$ & Lernprojekte & $\begin{array}{l}\text { Biografie, sozial-kulturelle } \\
\text { und sozial-ökologische Ein- } \\
\text { bettungen von Lernprozessen }\end{array}$ \\
\hline
\end{tabular}

- die Eigenstrukturen von Wissenschaft,

- die spezifische Strukturlogik explizit arrangierter Lehr-Lern-Situationen,

- fach-, studiengang- und standortbezogene [Lehr-]Kulturen,

- vielfältige idiosynkratische Lernhabitus,

- biografisch bedingten Eigen-Sinn sowie spezifisch individuelle Motivstrukturen.

Das Ziel dieser Explikation war, Lehren und Lernen an Hochschulen als Gegenstand der Bildungswissenschaft zunächst systematisch theoretisch zu erschließen.

Wodurch genau sich die unterschiedlichen Gestalten des Lernens an Hochschulen empirisch auszeichnen, ist dann eine Frage (rekonstruktiver) bildungswissenschaftlicher Hochschulforschung.

Leitfragen könnten dabei sein:

- Auf welche Weise eignen sich Studierende im Laufe ihres Studiums die durch die jeweiligen Fachwissenschaften artikulierten Wissens- und Könnensordnungen an? Welche Idiosynkrasien sind hier identifizierbar?

- In welchem Ausmaß erfolgt das Lernen im Studium in direkter Reaktion auf die (einschränkenden oder eröffnenden) fremd-arrangierten Gelegenheitsstrukturen des Studiums? Unter welchen Bedingungen entwickeln Studierende eigene Fragestellungen und Erkenntnisinteressen?

- Inwiefern gelingt es Studierenden, in der Auseinandersetzung mit disziplinären Imperativen Sinn- und Handlungsressourcen zu erschließen und das Studium als subjektiv bedeutsamen biografischen Abschnitt zu gestalten?

- Welche Optionen der Teilhabe an Wissensgemeinschaften, communities of practice oder akademisierten Handlungsfeldern eröffnen sich Studierenden? Und welche Teilhabeoptionen können sie sich aktiv erschließen? 
In spezifisch erwachsenenbildungswissenschaftlicher Perspektive lässt sich $u$. a. nach dem „Bildungswert“ von Wissenschaft sowie nach der Funktion von Hochschulen im Bildungssystem (auch im Kontext Lebenslangen Lernens) fragen:

Was genau bedeutet es, etwas zu wissen? Welche Funktion hat (wissenschaftliches bzw. an Wissenschaft orientiertes) Wissen in der reflexiven Moderne? Was lässt sich sinnvollerweise unter dem Begriff ,,wissenschaftliche Bildung“ in einer „Wissensgesellschaft" verstehen?

Welche Bildungsbedürfnisse und welche Bildungsbedarfe individueller wie gesellschaftlicher Art können Hochschulen befriedigen - insbesondere durch

- die Erzeugung eines technologischen, kulturellen, sozialen oder ökonomischen, aber auch epistemischen Mehrwertes, der sich mit der Ausübung bzw. Anwendung von Wissenschaft erzielen lässt,

- die Eröffnung tätigkeitsbezogener Anschlussoptionen, ${ }^{34}$

- die Verfeinerung von Fähigkeiten und Motivation zum aktiven Handeln in der demokratisch verfassten Gemeinschaft (,_citizenship““35)?

Noch spezifischer lässt sich, daran anschließend, fragen, wie erwachsenengerechte Lern- und Bildungsräume an der Hochschule (in normativer Hinsicht) beschaffen sein sollten und wie ,pädagogisch“ Hochschullehre sein darf bzw. muss.

Hochschuldidaktik schließlich lässt sich als Reflexions- und Beratungsform verstehen, die Orte generiert, an denen lehrpraktische Anliegen artikuliert sowie Anforderungen und Handlungsoptionen in der Gestaltung von Lehr-Lern-Situationen durchgearbeitet werden können.

Hochschuldidaktik erschöpft sich dabei weder in einer rhetorischen (Orientierung an Verständlichkeit) noch in einer pädagogischen Funktion (Orientierung am Lernen bzw. an den Lernenden). Hochschuldidaktik kann es als ihre Aufgabe ansehen, die Komplexität von Lehre in ihren Voraussetzungen und kontingenten, adressaten- und kontextsensiblen Gelingensbedingungen theoretisch zu modellieren und Lehrende durch fallrekonstruktive Reflexion ihres Lehrhandelns zu begleiten.

Theoretische Hochschuldidaktik zum praktischen Zwecke bedeutet

- das Zurückdenken auf den Eigen-Sinn von Wissenschaft im Allgemeinen bzw. der einzelnen Disziplinen im Besonderen mit ihren Anschluss- und Verwendungsoptionen;

- das Zurückdenken auf Ziel und Zweck der lernenden Auseinandersetzung mit Wissenschaft als Gegenstand des Studiums - sowohl aus der Perspektive der wissenschaftlichen Fächer in Bezug auf die Funktion eines Studiums als auch aus der Perspektive der Studierenden in Bezug auf die Erschließung von Sinn- und Handlungsressourcen;

- die Spezifikation der durch die Studierenden zu erbringenden akademisch-intellektuellen Leistungen - im Nach-Vollzug der Eigen-Logik von Wissenschaft und im Vollzug der Erschließung von Sinn- und Handlungsressourcen;

\footnotetext{
${ }^{34} \mathrm{Vgl}$. exemplarisch zum Verhältnis von „Wissenschaftlicher Rationalität und berufspraktischer Erfahrung“ Wittpoth 1987, zur Konfiguration von „Aufklärung, Wissenschaft und lebensentfaltender Bildung“ Faulstich 2011a.
}

${ }^{35}$ Vgl. Sliwka 2009, S. 119. 
- die Konzeptualisierung geeigneter Lernräume, in denen Studierende die notwendigen Lern- und Bildungsprozesse vollziehen können.

Hochschuldidaktik - in spezifisch erwachsenenbildungswissenschaftlicher Perspektive - fragt außerdem, wie sich theoretisches, wissenschaftliches Wissen zu beruflichem Wissen, Erfahrungswissen und lebensweltlicher Praxis verhält und welche subjektive Bedeutung abstraktes, wissenschaftliches Wissen gewinnen kann. ${ }^{36}$

Open Access Dieser Artikel wird unter der Creative Commons Namensnennung 4.0 International Lizenz (http://creativecommons.org/licenses/by/4.0/deed.de) veröffentlicht, welche die uneingeschränkte Nutzung, Verbreitung und Wiedergabe für beliebige Zwecke erlaubt, sofern Sie den/die ursprünglichen Autor(en) und die Quelle ordnungsgemäß nennen, einen Link zur Creative Commons Lizenz beifügen und angeben, ob Änderungen vorgenommen wurden.

\section{Literatur}

Conein, S., Schrader, J., \& Stadler, M. (Hrsg.). (2004). Erwachsenenbildung und die Popularisierung von Wissenschaft. Bielefeld: W. Bertelsmann.

Faulstich, P. (2008). Vermittler wissenschaftlichen Wissens. Bielefeld: Transcript.

Faulstich, P. (2011a). Aufklärung, Wissenschaft und lebensentfaltende Bildung. Bielefeld: Transcript.

Faulstich, P. (2011b). Übergänge, Öffnung, Durchlässigkeit, Anerkennung, Anrechnung, Passagen. Hessische Blätter für Volksbildung, 61(3), 203-205.

Friebertshäuser, B. (2004). Hochschulforschung. In H.-H. Krüger \& C. Grunert (Hrsg.), Wörterbuch Erziehungswissenschaft (S. 244-249). Wiesbaden: Verlag für Sozialwissenschaften.

Göhlich, M. (2011). Institutionen und Organisationen. In J. Kade, et al. (Hrsg.), Pädagogisches Wissen (S. 93-101). Stuttgart: Kohlhammer.

Göhlich, M., \& Zirfas, J. (2007). Lernen. Ein pädagogischer Grundbegriff. Stuttgart: Kohlhammer.

Habermas, J. (1968). Erkenntnis und Interesse. In J. Habermas (Hrsg.), Technik und Wissenschaft als „Ideologie“(S. 146-168). Frankfurt a. M.: Suhrkamp.

Heckhausen, H. (1987). „Interdisziplinäre Forschung“ zwischen Intra-, Multi- und Chimären-Disziplinarität. In J. Kocka (Hrsg.), Interdisziplinarität (S. 129-145). Frankfurt a. M.: Suhrkamp.

Helsper, W. (2011). Lehren. In J. Kade, et al. (Hrsg.), Pädagogisches Wissen. Erziehungswissenschaft in Grundbegriffen (S. 125-132). Stuttgart: Kohlhammer.

Hillmann, K.-H. (2007). Wörterbuch der Soziologie (5. Aufl.). Stuttgart: Alfred Kröner.

Hoyningen-Huene, P. (2009). Systematizität als das, was Wissenschaft ausmacht. Information Philosophie, 37(1), 22-27.

Hoyningen-Huene, P. (2013). Systematicity. The nature of science. New York: Oxford University Press.

Huber, L. (Hrsg.). (1984). Ausbildung und Sozialisation in der Hochschule. Enzyklopädie Erziehungswissenschaft (Bd. 11). Stuttgart: Klett-Cotta.

Kerres, M., Hanft, A., Wilkesmann, U., \& Wolff-Bendik, K. (Hrsg.). (2012). Studium 2020: Positionen und Perspektiven zum Lebenslangen Lernen an Hochschulen. Münster $\mathrm{u}$. a: Waxmann.

Koch, H.-A. (2008). Die Universität. Geschichte einer europäischen Institution. Darmstadt: WBG.

Krüger, W. (1984). Wissenschaftliche Weiterbildung. Bad Heilbrunn: Klinkhardt.

Luhmann, N. (1975). Macht. Stuttgart: Enke.

Marquard, O. (1981). Frage nach der Frage, auf die die Hermeneutik die Antwort ist. Philosophisches Jahrbuch, 88, 1-19.

Mattern, J. (1996). Ricoer. Hamburg: Junius.

\footnotetext{
${ }^{36}$ Hochschuldidaktische Reflexionen weisen dann Bezüge zu Theoriebeständen der Wissenschaftssoziologie auf, sofern diese die Vielfalt von Wissensformen und die Funktion und Bedeutung wissenschaftlichen Wissens in Bezug auf lebensweltliche Praxen untersucht. Vgl. einführend Weingart 2003; zur Konfiguration von „Aufklärung, Wissenschaft und lebensentfaltender Bildung“ Faulstich 2011a.
} 
Mecheril, P., \& Klingler, B. (2010). Universität als transgressive Lebensform. In L. Darowska, T. Lüttenberg, \& C. Machold (Hrsg.), Hochschule als transkultureller Raum? Kultur, Bildung und Differenz in der Universität (S. 83-116). Bielefeld: Transcript.

Pazzini, K.-J., Schuller, M., \& Wimmer, M. (Hrsg.). (2010). Lehren bildet? Vom Rätsel unserer Lehranstalten. Bielefeld: Transcript.

Schnädelbach, H. (1977). Reflexion und Diskurs. Fragen einer Logik der Philosophie. Frankfurt a. M.: Suhrkamp.

Sliwka, A. (2009). Citizenship. In S. Andresen, et al. (Hrsg.), Handwörterbuch Erziehungswissenschaft (S. 119-133). Weinheim: Beltz.

Stichweh, R. (1994). Wissenschaft, Universität, Profession. Frankfurt a. M.: Suhrkamp.

Stichweh, R. (2006). Die Universität in der Wissensgesellschaft. Wissensbegriffe und Umweltbeziehungen der modernen Universität. Soziale Systeme, 12(1), 33-53.

Teichler, U. (2004). Hochschule. In H.-H. Krüger \& C. Grunert (Hrsg.), Wörterbuch Erziehungswissenschaft (S. 239-244). Wiesbaden: Verlag für Sozialwissenschaften.

Teichler, U. (2009): Hochschulbildung. In R. Tippelt (Hrsg.), Handbuch der Bildungsforschung (S. 421444). 2. überarb. Aufl. Wiesbaden: Verlag für Sozialwissenschaften.

Teichler, U., \& Tippelt, R. (Hrsg.). (2005). Hochschullandschaft im Wandel. Zeitschrift für Pädagogik, 50. Beiheft.

Weber, W. E. J. (2002). Geschichte der europäischen Universität. Stuttgart: Kohlhammer.

Weingart, P. (2003). Wissenschaftssoziologie. Bielefeld: Transkript.

Wittpoth, J. (1987). Wissenschaftliche Rationalität und berufspraktische Erfahrung. Bad Heilbrunn: Klinkhardt.

Wolter, A. (2010). Die Hochschule als Institution des lebenslangen Lernens. In A. Wolter, G. Wiesner, \& C. Koepernik (Hrsg.), Der lernende Mensch in der Wissensgesellschaft: Perspektiven lebenslangen Lernens (S. 53-80). Weinheim: Juventa. 